\title{
CD7 promotes extramedullary involvement of the B-cell acute lymphoblastic leukemia line Tanoue by enhancing integrin $\beta 2$-dependent cell adhesiveness
}

\author{
TAKASHI KONDOH, KAGEAKI KURIBAYASHI, MAKI TANAKA, DAISUKE KOBAYASHI, \\ NOZOMI YANAGIHARA and NAOKI WATANABE
}

Department of Clinical Laboratory Medicine, Sapporo Medical University School of Medicine, Sapporo 060-8543, Japan

Received January 24, 2014; Accepted March 2, 2014

DOI: $10.3892 /$ ijo.2014.2492

\begin{abstract}
Extramedullary involvement (EMI) is a factor that defines prognosis of acute lymphoblastic leukemia; however, the molecular mechanism(s) remain elusive. Here, we show that CD7 promotes EMI of the human B-cell acute lymphoblastic leukemia cell line Tanoue. The Tanoue cell line expressing firefly luciferase, Luc-Tanoue, was transplanted into non-obese diabetic/severe combined immunodeficient mice, and cells infiltrated into the brain were cultured ex vivo. This process was repeated 4 times to obtain the highly invasive line Luc-Tanoue-F4. Comparison of the global gene expression signatures of Luc-Tanoue-F4 and Luc-Tanoue indicated that the $C D 7$ gene showed the largest increase in expression among EMI-related genes in Luc-Tanoue-F4 cells. Overexpression of CD7 in Tanoue enhanced cell invasiveness. Among cell migration, proliferation, adhesion and protease activity, only cell adhesiveness showed enhancement in Luc-Tanoue-F4. Expression of the intracellular domain, but not the extracellular domain, of CD7 enhanced cell adhesiveness. Luc-Tanoue-F4 showed a higher level of integrin $\beta 2$ expression; overexpression of CD7 induced the expression of integrin $\beta 2$ in Luc-Tanoue. These results show that CD7 induces integrin $\beta 2$ and enhances cell adhesiveness and invasiveness in Tanoue cells. This study highlights the role of the CD7/integrin $\beta 2$ axis as a critical pathway in the process of EMI of human B-cell acute lymphoblastic leukemia.
\end{abstract}

\section{Introduction}

Acute lymphoblastic leukemia (ALL), a malignant disorder of lymphoid progenitor cells, arises mainly in children and

Correspondence to: Professor Naoki Watanabe, Sapporo Medical University Hospital, Department of Clinical Laboratory Medicine, Sapporo Medical University, School of Medicine, South-1, West-16, Chuo-ku, Sapporo 060-8543, Japan

E-mail:watanabn@sapmed.ac.jp

Key words: acute lymphoblastic leukemia, extramedullary involvement, CD7, cell invasion, cell adhesion adolescents, and is the most common malignancy in these generations (1). Despite recent advances in the treatment of ALL, $20 \%$ of the patients cannot be cured even for those types of ALL with the best prognosis (2). Older age, higher leukocyte count, hypodiloidy, $t(9 ; 22), t(4 ; 11)$, and extramedullary involvement $(\mathrm{EMI})$ are the factors that relate to poor prognosis $(3,4)$. EMI not only associates with poor prognosis but also induces painful symptoms. At initial diagnosis, approximately 30-50, $8,2.5-5$ and $0.6 \%$ of patients show infiltration of leukemic cells into the liver, mediastinum, central nervous system (CNS), and testis, respectively (4-6). Moreover, solitary CNS or testicular relapse is experienced in 20.9 or $5.3 \%$ of relapsed patients, respectively (7). The T-cell immunophenotype, hyperleucocytosis, the Philadelphia reciprocal translocation between chromosome 9 and 22, and the presence of leukemic cells in the cerebrospinal fluid are factors that predict extramedullary relapse $(3,5,7)$.

ALL is a clonal disorder that is characterized by heterogeneous subpopulations of cells with different malignant behavior. The dissemination of leukemic cells (also referred to as metastasis in the context of solid tumors) is not a random event. Instead, this is a process destined by characteristic molecular events, with certain tumor cells having a specific affinity for the microenvironment $(8,9)$. In this context, significant effort has been devoted to finding molecules that direct leukemic cells to extramedullary organs. Clinically, overexpression of CXCR4, interleukin-15, CCR9, CD56, CD103, matrix metalloproteinase-2 (MMP-2), and MMP-9, or underexpression of intracellular adhesion molecule 1 (ICAM1) have been correlated with EMI (8,10-15). Experimental evidence suggested that Notch1 controls CCR7 expression and guides leukemic cells to CNS in vivo (16). Mass spectrometry revealed that leukemic cell lines with a higher invasiveness expressed RAC2 (17). Recently, Castro et al demonstrated that 5T4 oncofetal antigen enhances invasiveness of leukemic cell lines in vitro and in vivo (18).

Although an increasing number of studies are reporting on searches for molecules that associate with EMI of ALL, no study has used global gene expression analysis to compare leukemic clones with high and low invasiveness derived from the same cell line. In this study, we used the B-ALL cell line Tanoue to obtain a cell line with high invasiveness by in vivo 
selection and compared the gene signature with the parental cells.

\section{Materials and methods}

Cell line. The human B lymphoblastic leukemia cell line Tanoue was obtained from the Riken BioResource Center (Tsukuba, Japan). Tanoue was cultured in RPMI-1640 (Lonza, Basel, Switzerland) supplemented with $10 \%$ heat-inactivated fetal bovine serum (FBS; Hyclone, Logan, UT, USA) at $37^{\circ} \mathrm{C}$ in a humidified atmosphere of $5 \% \mathrm{CO}_{2}$.

Retroviral transfection of luciferase gene. Expression of the firefly luciferase gene was carried out as described, previously (19). Briefly, the retrovirus vector pBABE-Luc-Hygro was transfected into the packaging cell line Platinum-A (Cell Biolabs Inc., San Diego, CA, USA) using Lipofectamine 2000 (Life Technologies Inc., Gaithersburg, MD, USA) according to the manufacturer's instructions. The supernatant was collected, and Tanoue was infected with the virus in the presence of $8 \mu \mathrm{g} / \mathrm{ml}$ hexadimethrine bromide (Sigma-Aldrich, St. Louis, MO, USA). Luciferase-expressing Tanoue (Luc-Tanoue) cells were selected by exposure to $250 \mu \mathrm{g} / \mathrm{ml}$ of hygromycin B (Wako Pure Chemical Industries, Tokyo, Japan) for 1 week.

Non-invasive in vivo imaging and selection of highly infiltrative leukemic cell line. The study protocol was approved by the Animal Ethics Committee of Sapporo Medical University (no. 09-003). The tail vein of NOD/SCID mice (female, 5-week old) were injected with $1 \times 10^{6}$ Luc-Tanoue in $0.1 \mathrm{ml}$ RPMI. Bioluminescence signals were monitored weekly using the IVIS Lumina II (Caliper Life Sciences, Hopkinton, MA, USA) after isoflurane anesthesia was administered to the animals. Before imaging, each mouse was injected with $0.15 \mathrm{ml}$ luciferin $(20 \mathrm{mg} / \mathrm{ml}$ potassium salt; Promega, Madison, WI, USA), intraperitoneally. To establish a highly infiltrative leukemic cell line, the mice were monitored until leukemic cells infiltrated the CNS. Thereafter, leukemic cells in the brain was harvested, their culture in vitro generated the line the Luc-Tanoue-F1. Repetition of this selection cycle 4 times generated Luc-Tanoue-F4 line.

In vitro cell invasion and migration assay. Cells were seeded in the upper chamber of BD BioCoat Matrigel Invasion Chamber $(8-\mu \mathrm{m}$ pore size; BD Bioscience, San Jose, CA, USA) at a density of $1 \times 10^{6}$ cells per well in $0.5 \mathrm{ml}$ RPMI-1640 with $0.1 \%$ FBS and $0.5 \mathrm{ml}$ of RPMI-1640 containing $1 \%$ FBS was added to the lower chamber. Plates were incubated for $48 \mathrm{~h}$ at $37^{\circ} \mathrm{C}$ in a humidified atmosphere $\left(5 \% \mathrm{CO}_{2}\right)$ and cells that migrated into the lower chamber were enumerated with an XE-5000 hemocytometer (Sysmex, Kobe, Japan). The percentage of invasive cells was expressed as a percentage of total cells.

Cell migration assay was performed as described above, except that the Transwell chamber $(8-\mu \mathrm{m}$ pore size; Corning, Corning, NY, USA) was used as the assay plate, and the plates were incubated for $24 \mathrm{~h}$.

Cell proliferation assay. Cells were seeded in 24-well plates at a density of $1 \times 10^{5}$ cells per well in triplicate. Plates were incu- bated at $37^{\circ} \mathrm{C}$ in a humidified atmosphere $\left(5 \% \mathrm{CO}_{2}\right)$ and cell numbers were determined using an XE-5000 hemocytometer (Sysmex) at the time-points indicated in each experiment.

In vitro cell adhesion assay. Flat-bottomed 96-well plates (Corning) were coated with Matrigel (BD Bioscience) diluted with RPMI-1640 to $100 \mathrm{ng} / \mu \mathrm{l}$, seeded with $2 \times 10^{5}$ cells, incubated for $2 \mathrm{~h}$ at $37^{\circ} \mathrm{C}$, washed twice before measuring the number of attached cells using the Cell Titer-Glo ${ }^{\mathrm{TM}}$ Luminescent Cell Viability assay (Promega), according to the manufacturer's instructions. The level of ATP-derived luminescent signal was measured using a Veritas ${ }^{\mathrm{TM}}$ Microplate Luminometer (Promega). Adhered cells are described as a percentage of total cells seeded.

Zymography. MMP-2 and MMP-9 activity of cultured media was assessed by gelatin zymogram (Life Technologies Inc.) according to the manufacturer's instructions. Briefly, cells were cultured in RPMI-1640 without FBS for $24 \mathrm{~h}$, after which $10 \mu \mathrm{l}$ of the media was diluted 1:1 with Tris-glycine SDS sample buffer and separated by electrophoresis on a 10\%-Novex Zymogram (gelatin) gel. The gels were incubated in Zymogram Renaturing buffer for $30 \mathrm{~min}$ at room temperature, equilibrated with Zymogram Developing buffer and incubated overnight at $37^{\circ} \mathrm{C}$. The gels were stained with Coomassie brilliant blue and MMP activity was detected as clear bands against a dark background.

Quantitative reverse transcriptase-polymerase chain reaction. Expression of $C D 7$ mRNA was determined by quantitative reverse transcriptase-polymerase chain reaction (RT-PCR). Total RNA was isolated using the RNeasy Mini Kit (Qiagen), according to the protocol provided by the manufacturer. The cDNA was synthesized using TaqMan Reverse Transcription reagents (Applied Biosystems, Foster City, CA, USA). The gene-specific primers and fluorescent hybridization probes used in the quantitative PCR were as follows: $C D 7$ forward primer, 5'-TCGGACACTGGCACCTACAC-3'; reverse primer, 5'-TGCCATCCTTGGGACTGTTC-3'; and probe, 5'-TGCC AGGCCATCACGGAGGTCAAT (TAMRA)-3'. Expression level of $C D 7$ was compared to the level of GAPDH, which was determined using the GAPDH control reagents (Applied Biosystems). For ITGA3 and ITGB2, the primers and probes were purchased from Applied Biosystems.

Flow cytometric analysis and cell sorting. Surface immunophenotyping and cell sorting was performed using the EPICS XL-MCL flow cytometer (Beckman Coulter, Fullerton, CA, USA) and FACS Aria II cell sorter (Becton-Dickinson). Fluorescein isothiocyanate (FITC)-conjugated anti-CD7 antibody (6603824) was purchased from Beckman Coulter.

Microarray analysis. Global gene expression profiling was carried out by Hokkaido System Science (Sapporo, Japan) using Agilent RNA Spike-In Kit for One color and Agilent SurePrint G3 Human GE 8x60K Microarrays following the Agilent one-color microarray-based gene expression analysis protocol (Agilent Technologies, Santa Clara, CA, USA). The slides were scanned with an Agilent Technologies Microarray Scanner and the image data was processed using Agilent 

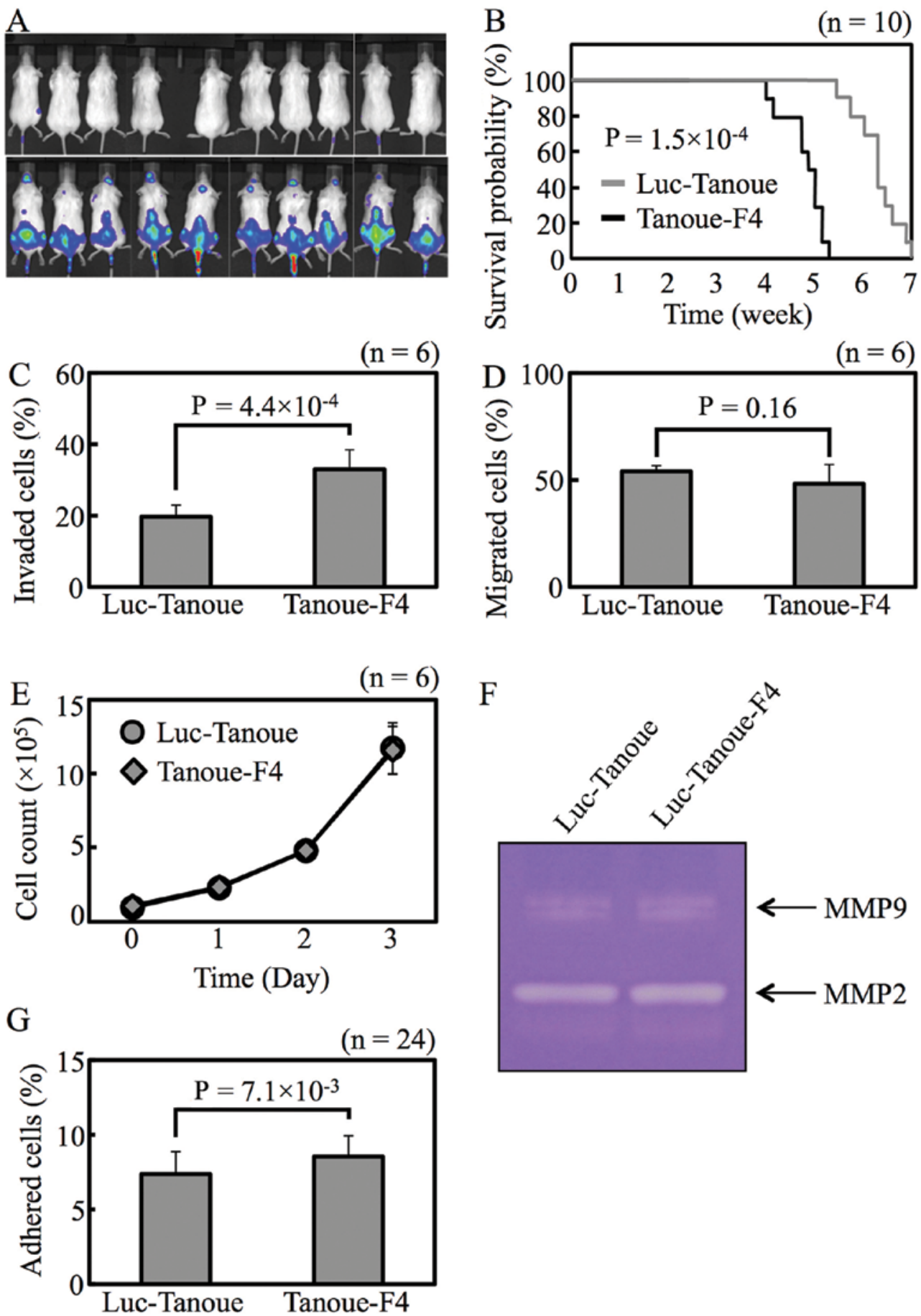

Figure 1. Selection of Luc-Tanoue-F4, a highly invasive cell line. (A) Luc-Tanoue (parental cells, upper panel) and Luc-Tanoue-F4 (a cell line obtained by in vivo selection, lower panel) were injected into the tail vein of each animal, and the bioluminescence signal was monitored every week. Images collected 2 weeks after injection are shown. The scales of luminescent pseudocolor image of upper and lower panel have been adjusted to indicate the same number of cells. (B) Survival curve of NOD/SCID mice injected with Luc-Tanoue and Luc-Tanoue-F4. (C) Matrigel invasion assay. (D) Transwell migration assay. (E) Cell proliferation assay. (F) Matrix metalloproteinase-2 and -9 activity assay by gelatin-zymography. (G) Matrigel cell adhesion assay. Error bars denote 2 standard deviations. P-values are obtained with unpaired Student's t-test.

Feature Extraction software, version 10.7.3.1. The gene expression levels were compared after global normalization.

Construction of CD7 expressing vectors and the transfection procedure. Full length $C D 7$ was amplified using 5'-GCTAG CAACATGGCCGGGCCTCCGAGGCTCC-3' and 5'-ACCG GTTGGTACTGGTTGGGGGAGGACAGC- 3 ' as the forward and reverse primer, respectively. Extracellular domain of $C D 7$ was amplified using 5'-GCTAGCAACATGGCCGGGCCT CCGAGGCTCC-3' and 5'-ACCGGTCTCGCCAGCACACA CGCCACCCC-3' as the forward and reverse primer, respectively. Intracellular domain of $C D 7$ was amplified using 5'-GCTAGCACCATGGCGAGGACACAGATAAAGAAAC-3' and 5'-ACCGGTTGGTACTGGTTGGGGGAGGACAGC-3' as the forward and reverse primer, respectively. The PCR products were cloned into PCR4-TOPO vector (Life Techno- 


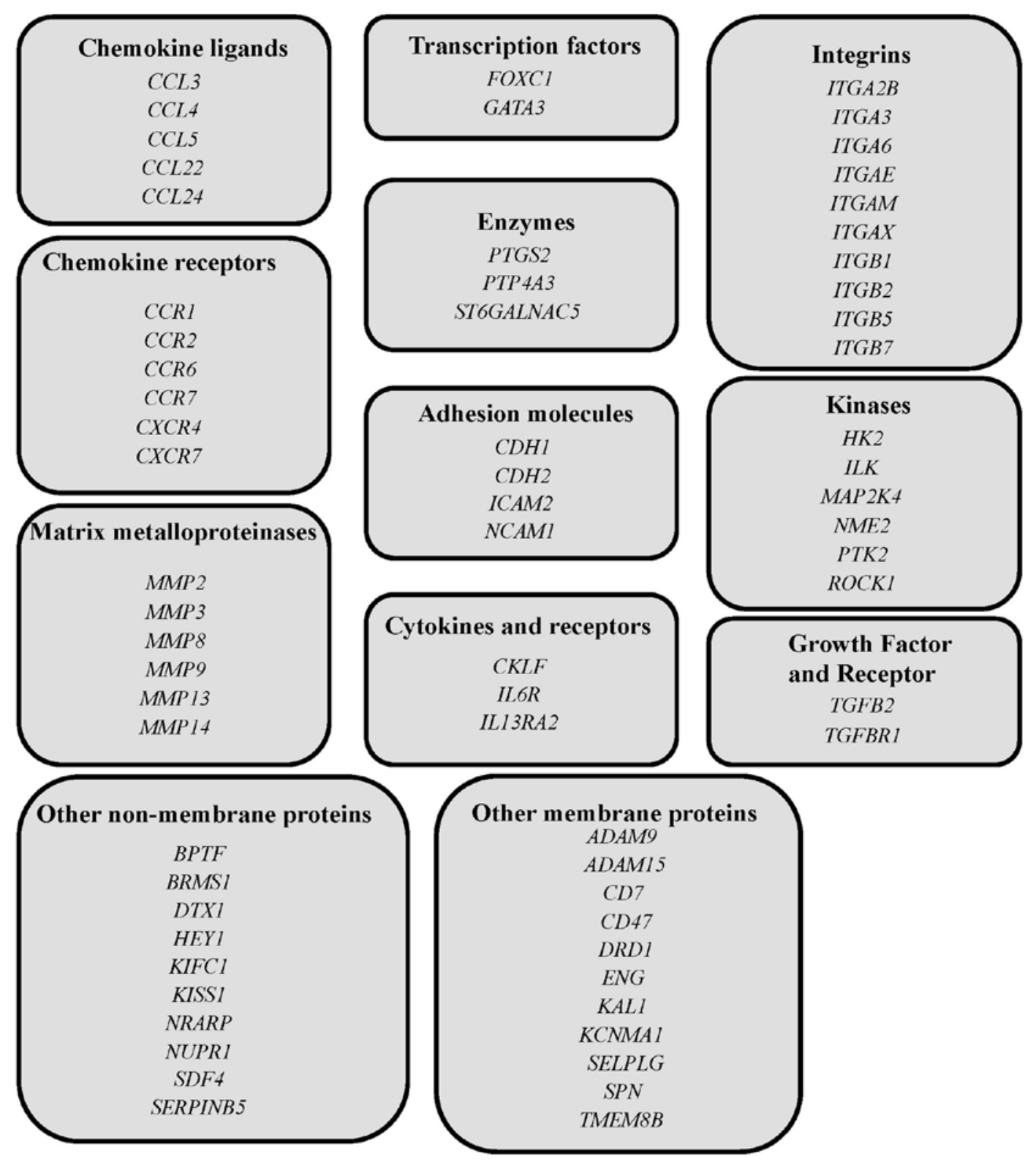

Figure 2. Genes related to extramedullary involvement or brain metastasis.

logies), sequenced, and the cDNA then excised by restriction digestion prior to cloning into the NheI and AgeI sites of pTurbo-GFP-N vector (Evrogen, Moscow, Russia), resulting in pTurboCD7-GFP, pTurboCD7-EC-GFP and pTurboCD7IC-GFP. The plasmids were transfected into cells using Lipofectamine 2000 (Invitrogen) according to the manufacturer's protocol. GFP-positive cells were selected using FACS Aria II cell sorter (Becton-Dickinson).

Silencing of CD7 and ITGB2 by short interfering RNA (siRNA. Stealth siRNAs against CD7 (HSS101524, HSS190108) and ITGB2 (HSS105563, HSS105564) were purchased from Life Technologies. Non-silencing siRNA control was purchased from Applied Biosystems. All siRNAs were transfected into cells by electroporation, using Amaxa cell line Nucleofector Kit T (Lonza, Gaithersburg, MD, USA) Nucleofector II (Lonza) and the program $\mathrm{C}-005$, according to the manufacturer's instructions.
Statistical analysis. Statistical analysis of the data was conducted using Microsoft Excel ${ }^{\circledR}$. Statistical significance was evaluated with the Student's t-test.

\section{Results}

Selection of a highly invasive leukemic cell line. The highly invasive cell line Luc-Tanoue-F4 was obtained by 4 rounds of in vivo selection. To confirm that Luc-Tanoue-F4 is more invasive than Luc-Tanoue, the cells were injected into the tail vein of non-obese diabetic/severe combined immunodeficient (NOD/SCID) mice, and EMI was monitored non-invasively in vivo. After 2 weeks, none of NOD/SCID mouse injected with Luc-Tanoue showed apparent CNS involvement; however, 9 of 10 mice injected with Luc-Tanoue-F4 revealed involvement of the brain or olfactory bulb (Fig. 1A). Mean survival was shorter in mice injected with Luc-Tanoue-F4 than Luc-Tanoue (Fig. $1 \mathrm{~B} ; 34.0 \pm 3.0$ vs. $44.0 \pm 3.4$ days, $\mathrm{P}=1.5 \times 10^{-4}$ ). 
A

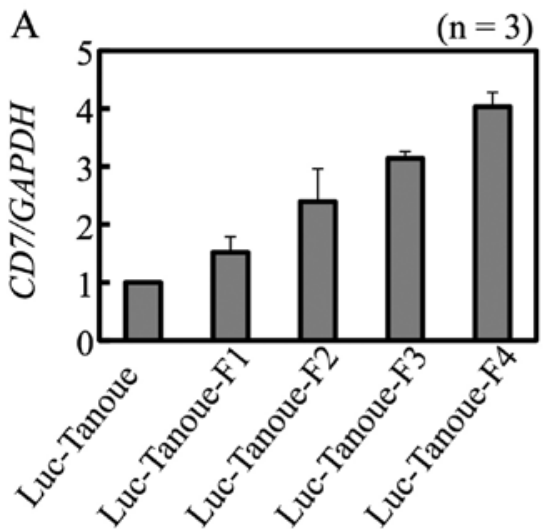

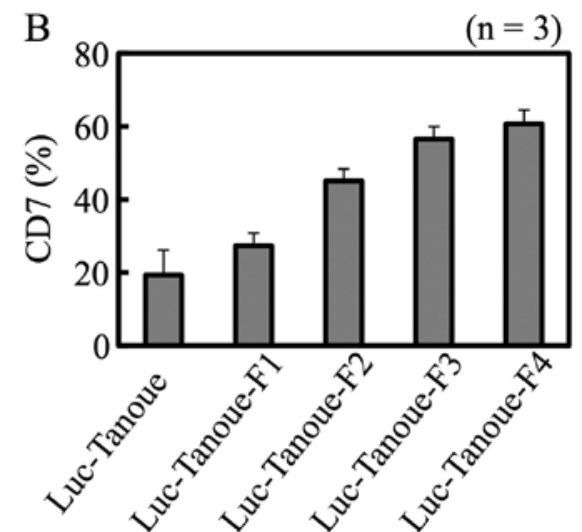

Figure 3. Expression of CD7 in Luc-Tanoue and Luc-TanoueF1-4 at the mRNA and protein levels. (A) Levels of CD7 mRNAs were quantified by TaqMan RT-PCR. Values denote relative expression after normalization with respect to GAPDH transcript levels. (B) Levels of CD7 proteins were determined by flow cytometry. All experiments were performed in triplicate and error bars denote 2 standard deviations.
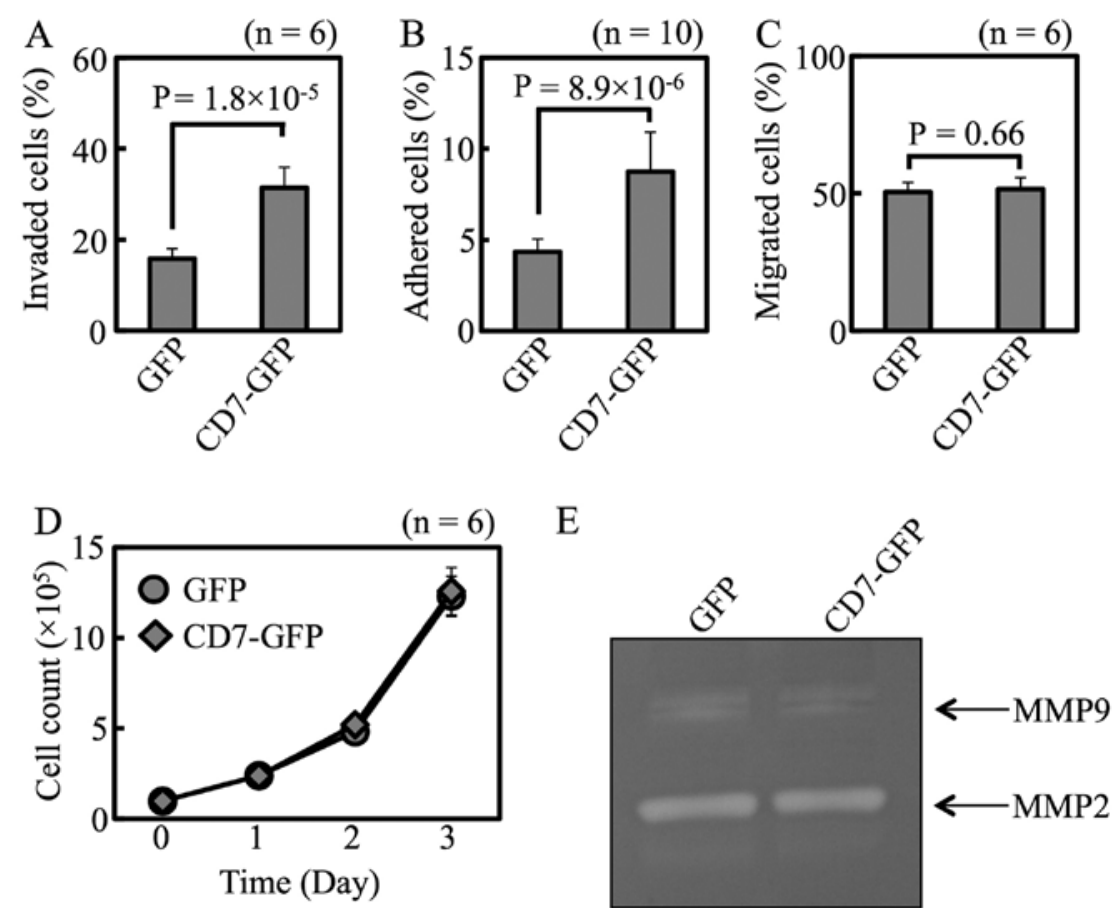

Figure 4. Stimulation of cell invasiveness and adhesion by overexpression of CD7. Tanoue cells transfected with pTurbo-GFP or pTurbo-CD7-GFP vector were selected in the presence of neomycin, and GFP-positive cells were collected by flow cytometry. (A) Matrigel invasion assay of pTurbo-GFP (left) and pTurbo-CD7-GFP (right) transfectants. (B) Matrigel cell adhesion assay using pTurbo-GFP (left) and pTurbo-CD7-GFP (right) transfectants. (C) Transwell migration assay of pTurbo-GFP (left) and pTurbo-CD7-GFP (right) transfectants. (D) Cell proliferation assay of pTurbo-GFP (left) and pTurbo-CD7-GFP (right) transfectants. (E) Gelatin zymography was assayed using pTurbo-GFP (left) and pTurbo-CD7-GFP (right) transfectants. Error bars denote 2 standard deviations. P-values are obtained with unpaired Student's t-test.

Luc-Tanoue-F4 was more invasive than Luc-Tanoue in vitro (Fig. 1C). Cell migration, proliferation, adhesion, and protease activity, all of which are the factors that affect cell invasiveness, were characterized in vitro. There were no differences between the 2 lines in terms of cell migration (Fig. 1D), proliferation (Fig. 1E), or protease activity (Fig. 1F). Cell adhesion was the only factor that differed between Luc-Tanoue and Luc-Tanoue-F4 (Fig. 1G).

CD7 expression is higher in Luc-Tanoue-F4 than Luc-Tanoue. Next, Luc-Tanoue and Luc-Tanoue-F4 were subjected to microarray analysis to compare gene-expression signatures.
When the cut-off value of gene expression level was set at 2-fold, 286 and 236 genes were expressed at higher and lower levels in Luc-Tanoue-F4 than Luc-Tanoue, respectively (GEO accession nos. GSE53651). Analysis of the expression of genes that relate to EMI or brain metastasis (listed in Fig. 2) indicated that $C D 7$ showed the largest difference (4.59-fold) in transcript levels between the 2 cell lines. Levels of ITGB2 (2.17-fold) and ITGA3 (2.13-fold) transcripts were also higher in Luc-Tanoue-F4 than Luc-Tanoue.

Levels of $C D 7$ transcripts in Luc-Tanoue and Luc-TanoueF1-F4 increased as the cells underwent in vivo selections (Fig. 3A). Analysis of CD7 protein abundance by fluorescence- 

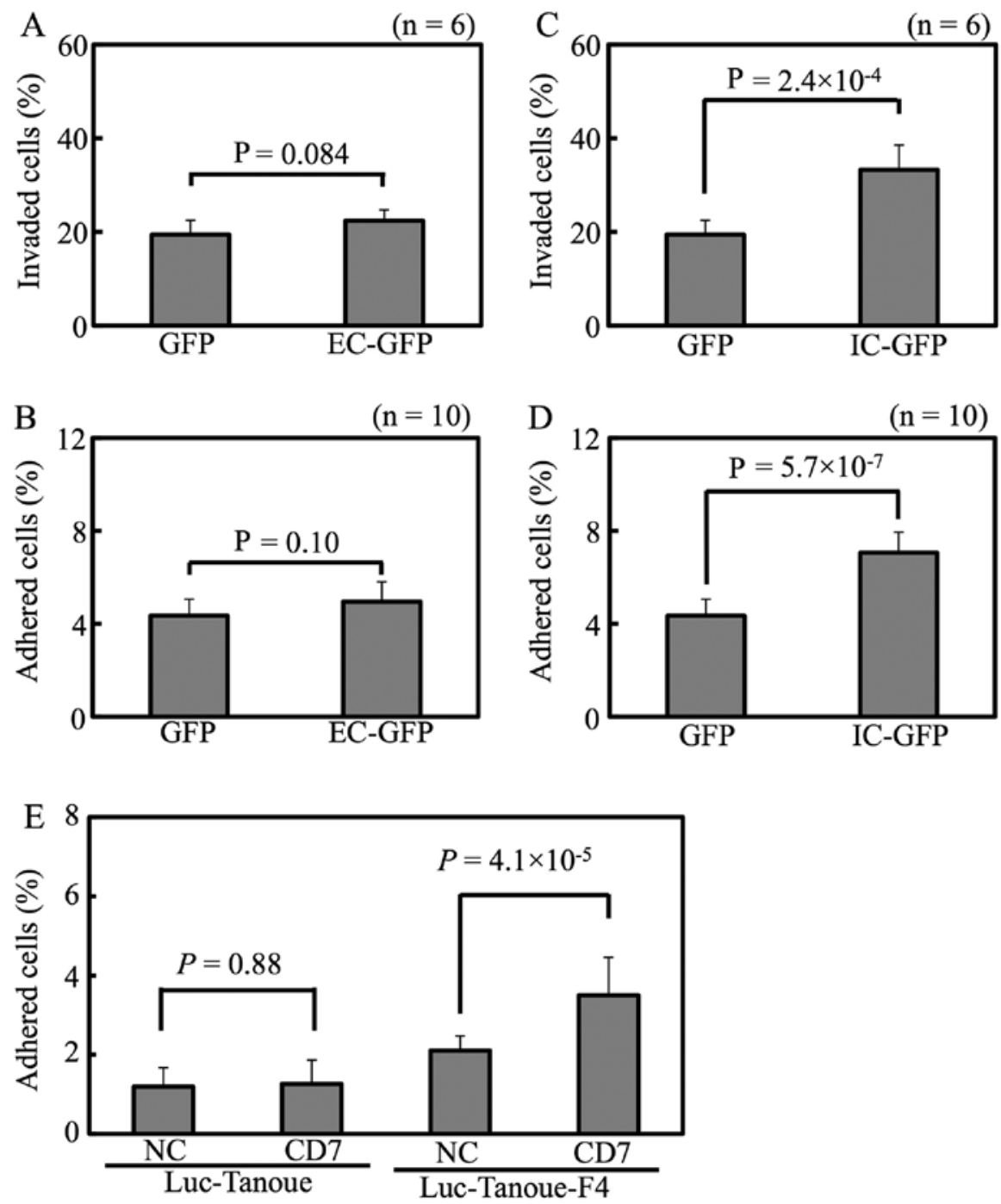

Figure 5. Analysis of Tanoue cells following their transfection with pTurbo-GFP, pTurbo-CD7-EC-GFP (a vector expressing extracellular domain of CD7 fused with GFP), or pTurbo-CD7-IC-GFP (a vector expressing intracellular domain of CD7 fused with GFP), selection in the presence of neomycin, and collection of GFP-positive cells by flow cytometry. (A) Matrigel invasion assay of Tanoue cells transfected with pTurbo-GFP (left) and pTurbo-CD7-EC-GFP (right). (B) Matrigel adhesion assay of Tanoue transfected with pTurbo-GFP (left) and pTurbo-CD7-EC-GFP (right). (C) Matrigel invasion assay of Tanoue transfected with pTurbo-GFP (left) and pTurbo-CD7-IC-GFP (right). (D) Matrigel adhesion assay of Tanoue transfected with pTurbo-GFP (left) and pTurbo-CD7-IC-GFP (right). Error bars denote 2 standard deviations. P-values were obtained with unpaired Student's t-test. (E) Matrigel cell adhesion assay of Luc-Tanoue and Tanoue-F4 in the presence of normal mouse IgG (NC, Beckman Coulter, A12689) or anti-CD7 agonistic antibody (CD7, Beckman Coulter, 6603824) (n=16). Error bars denote 2 standard deviations. P-values were obtained with unpaired Student's t-test.

activated cell sorting (FACS) was consistent with the result of gene expression analysis (Fig. 3B).

CD7 promotes cell invasiveness and adhesion. The CD7 protein was overexpressed as a fusion to green fluorescent protein (GFP) in Luc-Tanoue, a line that expresses CD7 at a low level. The $C D 7$ mRNA expression level was 51.0-fold higher in CD7-GFP transfectant than in the mock-transfected cells.

When measured in vitro, both cell invasiveness (Fig. 4A) and adhesion (Fig. 4B) were higher in CD7-GFP transfectants than mock-transfectants. There were no differences in cell migration, proliferation, or protease activity between these 2 transfectants (Fig. 4C-E).

These transfectants failed to express the GFP-fusion protein in vivo. Thus, Luc-Tanoue was separated into CD7-low expressing cells (Tanoue-CD $7_{\text {low }}$ ) and CD7-high expressing cells (Tanoue- $\mathrm{CD} 7_{\text {high }}$ ) by FACS. Tanoue-CD $7_{\text {high }}$ showed higher levels of invasiveness and cell adhesion than Tanoue$\mathrm{CD} 7_{\text {low }}$ in vitro (data not shown). Whereas all mice injected with Tanoue-CD $7_{\text {high }}$ showed olfactory bulb involvement, mice injected with Tanoue-CD7 $7_{\text {low }}$ showed no apparent EMI (data not shown). These results show that CD7 enhances the invasiveness of Tanoue cells in vitro and in vivo.

CD7 intracellular domain promotes cell invasiveness and adhesion. To gain insight into the mechanism by which CD7 enhances the adhesion of leukemic cells, the intracellular and extracellular domains of $C D 7$ were overexpressed separately in Luc-Tanoue. Whereas expression of the extracellular domain neither enhanced cell invasiveness (Fig. 5A) nor adhesiveness (Fig. 5B), the intracellular domain enhanced both cell invasiveness (Fig. 5C) and adhesiveness (Fig. 5D). Given the lack of availability of an anti-CD7 blocking antibody, Luc-Tanoue and Luc-Tanoue-F4 were stimulated with CD7-agonistic antibody; 

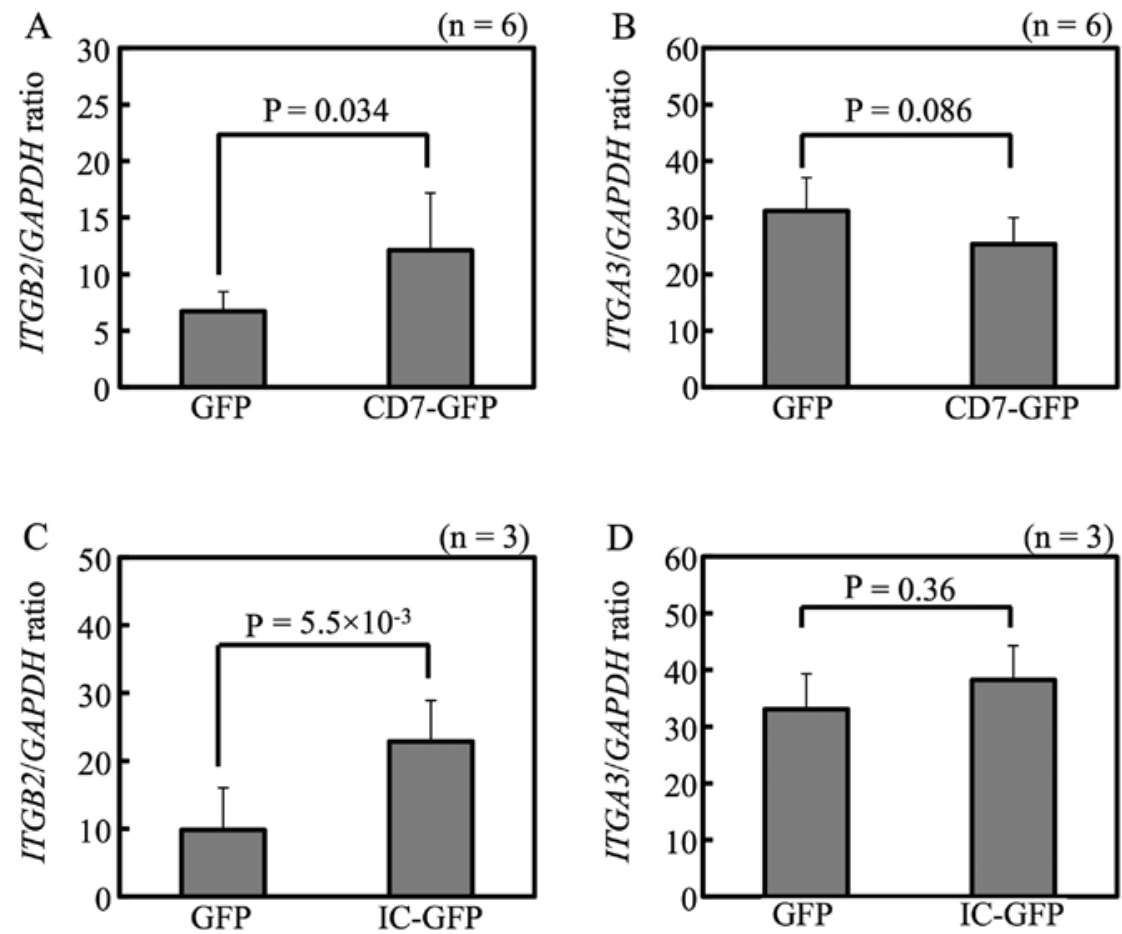

Figure 6. Induction of $I T G B 2$ by CD7. Levels of $I T G B 2$ (A and C) and ITGA3 (B and D) mRNAs were quantified by TaqMan RT-PCR following transfection of Tanoue cells with pTurbo-GFP-N, pTurboCD7-GFP and pTurboCD7-IC-GFP. Levels of target mRNAs were normalized relative to those of GAPDH transcripts. Error bars denote 2 standard deviations. P-values were obtained with unpaired Student's t-test.
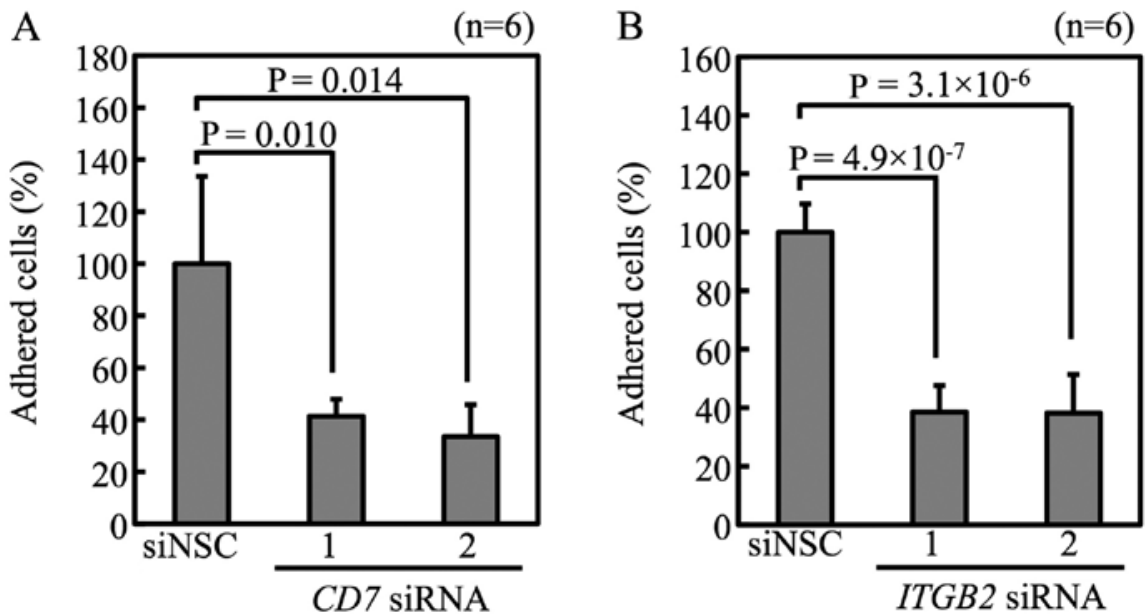

Figure 7. Matrigel cell adhesion assay of Luc-Tanoue-F4 transfected with non-silencing control siRNA (siNSC) or siRNA against CD7 (A) and ITGB2 (B). Error bars denote 2 standard deviations. P-values were obtained with unpaired Student's t-test.

whereas the CD7-agonistic antibody enhanced adhesion of the Luc-Tanoue-F4 (which expresses a high level of CD7), it had little effect on the Luc-Tanoue (which expresses a low level of CD7) (Fig. 5E). These results show that signal transduction evoked by the intracellular domain of CD7 enhanced the cell invasiveness and adhesive capacity of Tanoue cells.

Integrin $\beta 2$ mediates cell adhesion of Tanoue. To check whether CD7 regulates expression levels of ITGB2 and ITGA3, pTurbo-GFP-N, pTurboCD7-GFP and pTurboCD7IC-GFP transfectants were analyzed. As shown in Fig. 6A and $\mathrm{C}$, pTurboCD7-GFP and pTurboCD7-IC-GFP transfectants showed higher levels of ITGB2 expression than
pTurboGFP-N transfectants. Transfection of pTurboCD7-GFP and pTurboCD7-IC-GFP did not increase ITGA3 expression (Fig. 6B and D). Expression level of integrin $\beta 2$ was higher in Luc-Tanoue-F4 than Luc-Tanoue, confirmed by FACS (data not shown). Suppression of $C D 7$ and ITGB2 by siRNA reduced adhesion of Luc-Tanoue-F4 (Fig. 7). These results show that CD7 and integrin $\beta 2$ regulate adhesiveness of the Tanoue leukemic cell line.

\section{Discussion}

The present study revealed that CD7 promotes EMI of the B-ALL line Tanoue. To the best of our knowledge, this is 
the first study to show that CD7 promotes EMI by inducing integrin $\beta 2$ in hematological malignancy. Comparison of the global gene expression profile of cells with different EMI potential that were all obtained from the same B-ALL cell line and found no significant increases in any of the genes previously associated with EMI, including $C X C R 4, I L-15, C C R 9$, CD103, CD56, MMP2 or MMP9; nor did it suggest that a decrease in ICAMI expression was associated with EMI.

CD7 is a $40-k D a$ type-I transmembrane single-chain glycoprotein that belongs to immunoglobulin (Ig) superfamily. It is expressed on thymocytes, T cells, and natural killer (NK) cells, as well as in subpopulation of early immature $\mathrm{B}$ and myeloid cells (20). The extracellular domain of CD7 shares homology with the variable region of Ig $\kappa$-chains and the $\gamma$-chains of T-cell receptors (21). It is thought that CD7 exists as a homodimer, and that cross-linking antibodies that recognize CD7 stimulates its downstream signaling (22). There is a YEDM motif in the intracellular domain of CD7, and phosphatidylinositol 3-kinase and tyrosine kinase play critical roles in its activation (22-25).

Physiologically, activation of CD7 augments IL-2 production by $\mathrm{T}$ cells (26), induces the production of granulocyte macrophage colony-stimulating factor by myeloid progenitor cells (27), stimulates IFN- $\gamma$ production in NK cells (28), and regulates the capacity for adhesion in T cells (29) and NK cells (28).

Clinically, the expression of CD7 correlates with a poor prognosis for acute myeloid leukemia (30-35), non-Hodgkin's lymphoma (36) and B-lymphoblastic leukemia (37). Moreover, $\mathrm{CD}^{+}, \mathrm{CD} 4-\mathrm{CD} 8$ acute lymphoblastic leukemia is reported to show poor clinical characteristics that involves extramedullary organs, including the mediastenum, skin, and CNS (38).

This study showed that CD7 induces integrin $\beta 2$ (also referred to as CD18; Fig. 6A) and enhanced the capacities of leukemic cells for adhesion (Fig. 4B). Notwithstanding, there are observations that $\mathrm{CD} 7$ regulates the capacities of $\mathrm{T}$ and NK cells to adhere to extracellular matrix, there is no evidence that CD7 plays an adhesion molecule role. Together with the results shown in Fig. 5A-D that CD7-induced signaling (but not extracellular domain of CD7) enhanced the adhesive capacities of Tanoue cells, it seems possible that the induction of integrin $\beta 2$ enables $\mathrm{CD} 7$ to promote cell adhesiveness in the Tanoue B-ALL line. These observations are consistent with previous reports that $\mathrm{CD} 7$-induced cell adhesion is mediated by integrin $\beta 2(29,39)$.

Integrin $\beta 2$ is an important adhesion molecule in leucocytes. Genetic mutations in ITGB2 results in the immunodeficiency caused by a decreased capacity of leucocytes to adhere (leukocyte adhesion deficiency) $(40,41)$. Furthermore, integrin $\beta 2$ is critically required for leucocyte extravasation $(42,43)$. Together with our results, these observations indicate that the $\mathrm{CD} 7 /$ integrin $\beta 2$ axis may contribute to extravasation during the EMI of leukemic cells.

Results of this study imply that CD7 and integrin $\beta 2$ are potential molecular targets in leukemia therapy. Indeed, antibodies against integrin $\beta 2$ inhibit leukemia and lymphoma dissemination in experimental models (44-46). It remains to be established whether a blocking antibody against CD7 can inhibit dissemination of leukemia.
In conclusion, $\mathrm{CD} 7$ promotes EMI of the B-ALL line Tanoue in an integrin $\beta 2$-dependent manner. CD7 and integrin $\beta 2$ are potential molecular targets in leukemia therapy.

\section{Acknowledgements}

We thank Dr S.H. Kim (National Cancer Center, Korea) for providing us with firefly luciferase expressing vector (pBABELuc-Hygro).

\section{References}

1. Pui $\mathrm{CH}$ and Evans WE: Acute lymphoblastic leukemia. N Engl J Med 339: 605-615, 1998.

2. Schrappe M, Nachman J, Hunger S, et al: 'Educational symposium on long-term results of large prospective clinical trials for childhood acute lymphoblastic leukemia (1985-2000)'. Leukemia 24: 253-254, 2010.

3. Pui CH, Robison LL and Look AT: Acute lymphoblastic leukaemia. Lancet 371: 1030-1043, 2008.

4. Schrappe M, Reiter A, Ludwig WD, et al: Improved outcome in childhood acute lymphoblastic leukemia despite reduced use of anthracyclines and cranial radiotherapy: results of trial ALL-BFM 90. German-Austrian-Swiss ALL-BFM Study Group. Blood 95: 3310-3322, 2000.

5. Lazarus HM, Richards SM, Chopra R, et al: Central nervous system involvement in adult acute lymphoblastic leukemia at diagnosis: results from the international ALL trial MRC UKALL XII/ECOG E2993. Blood 108: 465-472, 2006.

6. Reiter A, Schrappe M, Ludwig WD, et al: Chemotherapy in 998 unselected childhood acute lymphoblastic leukemia patients. Results and conclusions of the multicenter trial ALL-BFM 86. Blood 84: 3122-3133, 1994.

7. Jacobs JE and Hastings C: Isolated extramedullary relapse in childhood acute lymphocytic leukemia. Curr Hematol Malig Rep 5: 185-191, 2010.

8. Annels NE, Willemze AJ, van der Velden VH, et al: Possible link between unique chemokine and homing receptor expression at diagnosis and relapse location in a patient with childhood T-ALL. Blood 103: 2806-2808, 2004.

9. Langley RR and Fidler IJ: Tumor cell-organ microenvironment interactions in the pathogenesis of cancer metastasis. Endocr Rev 28: 297-321, 2007.

10. Crazzolara R, Kreczy A, Mann G, et al: High expression of the chemokine receptor CXCR4 predicts extramedullary organ infiltration in childhood acute lymphoblastic leukaemia. Br J Haematol 115: 545-553, 2001.

11. Kuittinen O, Savolainen ER, Koistinen P, Mottonen M and Turpeenniemi-Hujanen T: MMP-2 and MMP-9 expression in adult and childhood acute lymphatic leukemia (ALL). Leuk Res 25: 125-131, 2001.

12. Mielcarek M, Sperling C, Schrappe M, Meyer U, Riehm H and Ludwig WD: Expression of intercellular adhesion molecule 1 (ICAM-1) in childhood acute lymphoblastic leukaemia: correlation with clinical features and outcome. Br J Haematol 96: 301-307, 1997.

13. Ravandi F, Cortes J, Estrov Z, et al: CD56 expression predicts occurrence of CNS disease in acute lymphoblastic leukemia. Leuk Res 26: 643-649, 2002.

14. Schneider P, Costa O, Legrand E, et al: In vitro secretion of matrix metalloproteinase-9 is a prognostic marker in childhood acute lymphoblastic leukemia. Leuk Res 34: 24-31, 2010.

15. Wu S, Fischer L, Gokbuget N, et al: Expression of interleukin 15 in primary adult acute lymphoblastic leukemia. Cancer 116: 387-392, 2010.

16. Buonamici S, Trimarchi T, Ruocco MG, et al: CCR7 signalling as an essential regulator of CNS infiltration in T-cell leukaemia. Nature 459: 1000-1004, 2009.

17. Holland M, Castro FV, Alexander S, et al: RAC2, AEP, and ICAM1 expression are associated with CNS disease in a mouse model of pre-B childhood acute lymphoblastic leukemia. Blood 118: 638-649, 2011.

18. Castro FV, McGinn OJ, Krishnan S, et al: 5T4 oncofetal antigen is expressed in high risk of relapse childhood pre-B acute lymphoblastic leukemia and is associated with a more invasive and chemotactic phenotype. Leukemia 26: 1487-1498, 2012. 
19. Kuribayashi K, Finnberg N and El-Deiry WS: Studying p53-dependent cell death in vitro and in vivo. Methods Enzymol 446: 159-173, 2008

20. Sempowski GD, Lee DM, Kaufman RE and Haynes BF Structure and function of the CD7 molecule. Crit Rev Immunol 19: 331-348, 1999.

21. Aruffo A and Seed B: Molecular cloning of two CD7 (T-cell leukemia antigen) cDNAs by a COS cell expression system. EMBO J 6: 3313-3316, 1987

22. Lazarovits AI, Osman N, Le Feuvre CE, Ley SC and Crumpton MJ: CD7 is associated with CD3 and CD45 on human T cells. J Immunol 153: 3956-3966, 1994.

23. Chan AS, Mobley JL, Fields GB and Shimizu Y: CD7-mediated regulation of integrin adhesiveness on human $T$ cells involves tyrosine phosphorylation-dependent activation of phosphatidylinositol 3-kinase. J Immunol 159: 934-942, 1997.

24. Lee DM, Patel DD, Pendergast AM and Haynes BF: Functional association of CD7 with phosphatidylinositol 3-kinase: interaction via a YEDM motif. Int Immunol 8: 1195-1203, 1996

25. Rabinowich H, Lin WC, Herberman RB and Whiteside TL: Signaling via CD7 molecules on human NK cells. Induction of tyrosine phosphorylation and beta 1 integrin-mediated adhesion to fibronectin. J Immunol 153: 3504-3513, 1994.

26. Jung LK, Roy AK and Chakkalath HR: CD7 augments T cell proliferation via the interleukin-2 autocrine pathway. Cell Immunol 141: 189-199, 1992.

27. Hou Z, Leta E and Jung LK: Cross-linking CD7 on myeloblasts results in granulocyte-macrophage colony-stimulating factor production. Blood 88: 124-129, 1996.

28. Rabinowich H, Pricop L, Herberman RB and Whiteside TL: Expression and function of CD7 molecule on human natural killer cells. J Immunol 152: 517-526, 1994.

29. Shimizu Y, van Seventer GA, Ennis E, Newman W, Horgan KJ and Shaw S: Crosslinking of the T cell-specific accessory molecules CD7 and CD28 modulates T cell adhesion. J Exp Med 175: 577-582, 1992.

30. Del Poeta G, Stasi R, Venditti A, et al: Prognostic value of cell marker analysis in de novo acute myeloid leukemia. Leukemia 8 : 388-394, 1994

31. Jensen AW, Hokland M, Jorgensen H, Justesen J, Ellegaard J and Hokland P: Solitary expression of CD7 among T-cell antigens in acute myeloid leukemia: identification of a group of patients with similar T-cell receptor beta and delta rearrangements and course of disease suggestive of poor prognosis. Blood 78: 1292-1300, 1991.

32. Kita K, Miwa H, Nakase K, et al: Clinical importance of CD7 expression in acute myelocytic leukemia. The Japan Cooperative Group of Leukemia/Lymphoma. Blood 81: 2399-2405, 1993.

33. Saxena A, Sheridan DP, Card RT, McPeek AM, Mewdell CC and Skinnider LF: Biologic and clinical significance of CD7 expression in acute myeloid leukemia. Am J Hematol 58 278-284, 1998.
34. Yumura-Yagi K, Hara J, Kurahashi H, et al: Clinical significance of CD7-positive stem cell leukemia. A distinct subtype of mixed lineage leukemia. Cancer 68: 2273-2280, 1991.

35. Chang H, Yeung J, Brandwein J and Yi QL: CD7 expression predicts poor disease free survival and post-remission survival in patients with acute myeloid leukemia and normal karyotype. Leuk Res 31: 157-162, 2007.

36. Yumura-Yagi K, Ishihara S, Hara J, et al: Poor prognosis of mediastinal non-Hodgkin's lymphoma with an immature phenotype of $\mathrm{CD} 2^{+}, \mathrm{CD} 7(\text { or } \mathrm{CD} 5)^{+}, \mathrm{CD} 3^{-}, \mathrm{CD} 4^{-}$, and $\mathrm{CD} 8$. Cancer 63 671-674, 1989.

37. Hussein S, Gill KZ, Sireci AN, et al: Aberrant T-cell antigen expression in B lymphoblastic leukaemia. Br J Haematol 155: 449-456, 2011.

38. Kurtzberg J, Waldmann TA, Davey MP, et al: $\mathrm{CD}^{+}, \mathrm{CD}^{-}$ CD8 acute leukemia: a syndrome of malignant pluripotent lymphohematopoietic cells. Blood 73: 381-390, 1989.

39. Chan AS, Reynolds PJ and Shimizu Y: Tyrosine kinase activity associated with the $\mathrm{CD} 7$ antigen: correlation with regulation of $\mathrm{T}$ cell integrin function. Eur J Immunol 24: 2602-2608, 1994.

40. Kishimoto TK, Hollander N, Roberts TM, Anderson DC and Springer TA: Heterogeneous mutations in the beta subunit common to the LFA-1, Mac-1, and p150,95 glycoproteins cause leukocyte adhesion deficiency. Cell 50: 193-202, 1987.

41. Mathew EC, Shaw JM, Bonilla FA, Law SK and Wright DA: A novel point mutation in CD18 causing the expression of dysfunctional CD11/CD18 leucocyte integrins in a patient with leucocyte adhesion deficiency (LAD). Clin Exp Immunol 121: 133-138, 2000.

42. Kling D, Fingerle J and Harlan JM: Inhibition of leukocyte extravasation with a monoclonal antibody to CD18 during formation of experimental intimal thickening in rabbit carotid arteries. Arterioscler Thromb 12: 997-1007, 1992.

43. Walzog B, Scharffetter-Kochanek K and Gaehtgens P: Impairment of neutrophil emigration in CD18-null mice. Am J Physiol 276: G1125-G11130, 1999.

44. Cohen S, Haimovich J and Hollander N: Anti-idiotype $x$ anti-LFA-1 bispecific antibodies inhibit metastasis of B cell lymphoma. J Immunol 170: 2695-2701, 2003.

45. Harning R, Myers C and Merluzzi VJ: Monoclonal antibodies to lymphocyte function-associated antigen-1 inhibit invasion of human lymphoma and metastasis of murine lymphoma. Clin Exp Metastasis 11: 337-342, 1993

46. Zahalka MA, Okon E and Naor D: Blocking lymphoma invasiveness with a monoclonal antibody directed against the beta-chain of the leukocyte adhesion molecule (CD18). J Immunol 150: 4466-4477, 1993. 